\title{
ETUDE DES SURCOTES DE MAREE DUES AU VENT
}

\author{
H. GHADRI ; O.A. KHALIFA M.A. ; A. GROVEL \\ Laboratoire de Mécanique et Géomécanique \\ Université de Nantes, 2 rue de la Houssinière - 44072 NANTES CEDEX
}

\begin{abstract}
On October 16 th, 1987, a $1.80 \mathrm{~m}$ surge (unforecast) was recorded in St-Nazaire with a 26 coefficient. Such a surge, combined with spring tide, would flood most of the Loire river-banks. It would endanger many urban areas and result in catastrophic ecological and economical consequences.
\end{abstract}

The present work is aimed at studying surges in atlantic coast. It is, above all. phenomenological and should lead to a bettre knowledge of surge forecast.

Tide-gauge data (every $5 \mathrm{mn}$ ) on the one hand, anemometric and barometric inshore and offshore data on the other hand, spanning the years 1985 to 1990 in StNazaire and St-Gildas, were compiled. The use of astronomical tide forecast. together with these experimental data, aliowed to gain a better understanding of wind influence on surge (positif or negatif).

Indead, we were able to isolate the wind direction and wind speed on the on hand, set-up winds on the other hand, and to analyse their respective effects.

\section{INTRODUCTION}

Lors de la violente tempête du 15 el ló Octobre 1987, une surcote de 1.80 mètre a été relévée à St-Nazaire par marée de morte eau de coefficient 26 . Une telle surcote qui surviendrait en conjonction avec une marée de vive-eau inonderait une grande partie des rives de la Loire. Elle mettrait en péril de nombreuses agglomérations urbaines et aurait des conséquences écologiques et économiques catastrophiques.

Les surcotes de marée sont la conséquence des conditions météorologiques sévères. La pression atmosphérique, la direction du vent et son intensité, le coefficient de marée astronomique, la morphologie côtière, ... sont autant de facteurs entrant en ligne de compte dans la formation de la surcote. La multitude de ces paramètres rend l'étude des surcotes complexe 
(GHADRI,1990,1991). Plusieurs études relatives à ce thème cherchent à cerner ce phénomène. Dans la plupart des cas, elles ont un caractère local. Souvent. elles portent sur des modèles numériques, statistiques ou probabilistes. En France, le LNH (EDF, Chatou) a réalisé un code de calcul simulant les surcotes en mer du Nord (DALSECO.1984). Une étude statistique des surcotes dans la Loire a été réalisée par ce même laboratoire dans le cadre du projet de centrale nucléaire du carnet (TEISSON, 1984). En Hollonde, un modèle probabiliste de prédiction de surcote a été élaboré par VRIJLING (1990) pour simuler les effets de tempêtes en mer du Nord et servir de base au dimensionnement des barrages anti-tempêtes.

Soulignons par ailleurs quil est aussi important de connaître les décotes que les surcotes de marée. En effet, celles-ci sont importantes dans le dimensionnement des ouvrages maritimes ainsi que pour la navigation.

Notre approche d'étude des surcotes est basée sur une meilleure compréhension du phénomène physique de surcote. par le traitement d'une importante quantité de données. Avant de procéder à la généralisation de l'étude des surcotes sur la facade atlantique, le site expérimental choisi est l'estuaire de la Loire, où nous disposons de mesures marégraphiques barométriques et anémométriques s'étalant sur une grande période.

Cette étude est encore en cours. Nous exposons dans ce document les conclusions qui nous paraissent les plus intéressantes et qui contribueront à mieux cerner les surcotes de marée.

Dans cet article, nous présentons d'abord les données utilisées dans l'étude avant de détailler la méthodologie adoptée. Enfin nous discuterons des résultats de ce travail ainsi que de ses développements en cours ou à réaliser.

\section{MATERIEL}

Plusieurs matériels ont été utilisés pour obtenir les données expérimentales:

1 - Deux marégraphes à flotteur, l'un mouillé au port de St-Nazaire, l'autre à la pointe de St-Gildas. Ces marégraphes sont équipés de système d'acquisition de données. Les marégrammes sont discrétisés dans le temps suivant un pas de $5 \mathrm{mn}$. Cet équipement est géré par le Port Autonome de Nantes-St-Nazaire.

2 - Matériels d'enregistrements anémométriques et barométriques de la station météorologique de laérodrome de GRON (Montoir de Bretagne). Cet éauibement fournit sur support informatisé des informations trihoraires 
concernant la pression atmosphérique ainsi que la vitesse et la direction du vent. Cet équipement est géré par Météo France.

3 - Mesures et observations de navires croisant au large de la façade atlantique. Les renseignements portent notamment sur les mesures de pression atmosphérique. de vitesse et de direction du vent. Les données que nous avons exploitées sont situées dans une zone géographique comprise entre $(46,5 \mathrm{~N}$ $47.5 \mathrm{~N}$ et $6 \mathrm{~W}-8 \mathrm{~W}$ ) présentée en figure 1.

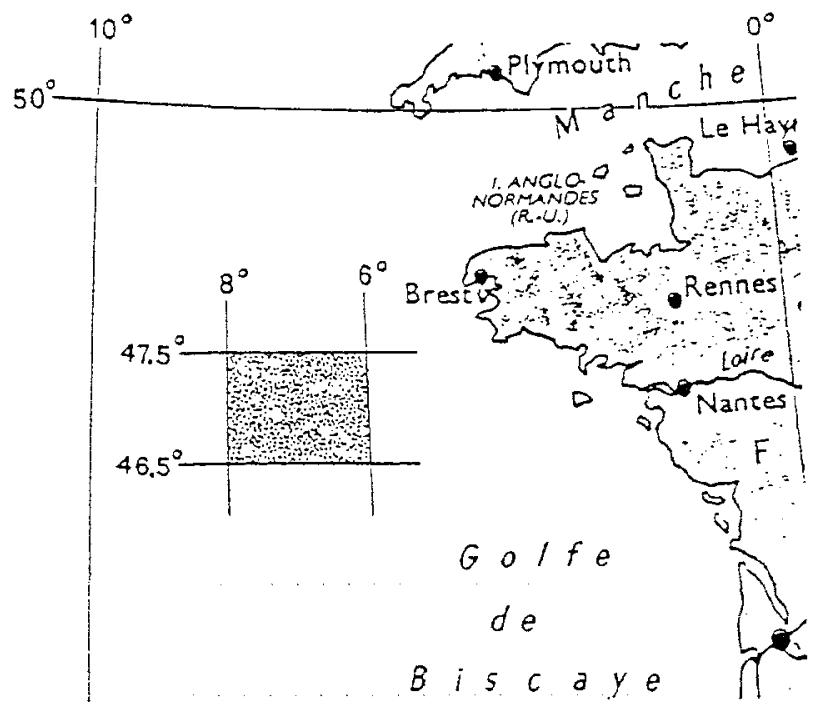

Fig. 1: Zone géographique des mesures de vent au large.

\section{METHODOLOGIE}

\section{1 - Principe}

Pour pouvoir calculer une surcote, il faut disposer d'une part d'enregistrements marégraphiques et d'autre part d'une méthode de prédiction de la marée astronomique.

- La méthode de prédiction la plus utilisée en france (SHOM) est celle de I'analyse harmonique (DOODSON, 1954). Cette méthode est basée sur la décomposition de l'onde marée enregistrée sur une longue période en une somme d'ondes élémentaires dont les périodes correspondent à des mouvements d'astres. 
- Les données expérimentales se rapportent aux données marégraphiques précédemment décrites à St-Nazaire et St-Gildas de 1985 à 1990 compris, et aux données barométriques et anémométriques, d'une part à l'aérodrome de GRON (Montoir de Bretagne) pendant la même durée, et d'autre part au large (Fig. 1) pendant la période comprise entre 01/01/85 et $31 / 12 / 89$.

\section{2 - Dépouillement}

Le dépouillement de ces données a nécessité l'élaboration du plusieurs programmes de calculs et a été fait en plusieurs étapes:

- Tri des fichiers de mesures afin de détecter les lacunes de mesures ainsi que les données abérrantes.

- Extraction des pleines mers et basses mers des fichiers de mesures et contrôles systématiques de celles-ci.

- Caiculs des pleines mers et basses mers prédites à St-Nazaire et StGildas.

- Calcuis des surcotes brutes (sans aucune correction).

Afin de disposer d'un outil de travail facilement exploitable, nous avons constitué une base de données comprenant:

- Les pleines mers et basses mers observées et prédites.

- Les coefficients de marée.

- La pression atmosphérique à Saint-Nazaire (côte).

- La vitesse et la direction du vent à St-Nazaire.

- La pression atmosphérique au large.

- La vitesse ef la direction du vent au large.

- La surcote corrigée de la pression (baromètre inversé).

Les données anémométriques et barométriques ont été préalablement interpolées aux instants de pleines mers et basses mers. Cette base de données globale contient plus de 70000 mesures marégraphiques. barométriques et anémométriques aux instants de pleines mers et basses mers.

\section{3- Traitement}

Afin d'étudier l'influence des paramètres du vent, nous avons cherché à les isoler et à mettre en exergue l'impact d'un seul paramètre. 
- Tri de la base de données globale suivant les revifs et déchets.

- Découpage des directions en 8 secteurs de $45^{\circ}$ centrés sur les directions (N, NE, E, SE, S, SN,W, NW)

- Découpage des vitesses de vent local en 4 plages correspondant aux forces de vent de l'échelle de BEAUFORT.

$$
\begin{array}{lll}
\text { V1 }: & 0 \text { a } 1.5 \mathrm{~m} / \mathrm{s} & \text { calme + très légère brise } \\
\text { v2 }: 1.6 \text { à } 5.4 \mathrm{~m} / \mathrm{s} & \text { légère brise + petite brise } \\
\text { V3 }: 5.5 \text { a } 10.7 \mathrm{~m} / \mathrm{s} & \text { jolie brise + bonne brise } \\
\text { V4 }: 10.8 \text { a } 17.1 \mathrm{~m} / \mathrm{s} & \text { vent frais + grand frais }
\end{array}
$$

- Découpage des vitesses de vent du large en 4 plages:

$$
\begin{aligned}
& \text { W1 : } 0 \text { à } 4.1 \mathrm{~m} / \mathrm{s} \\
& \text { W2 } 14.2 \text { à } 14.6 \mathrm{~m} / \mathrm{s} \\
& \text { W3 }: 14.7 \text { à } 28.9 \mathrm{~m} / \mathrm{s} \\
& \text { W4: } 29.0 \text { à } 46.0 \mathrm{~m} / \mathrm{s}
\end{aligned}
$$

- Tri de la base de données globale suivant les 8 secteurs de directions et constitution de 8 bases de données partielles.

- Tri de chacune des bases de données partielles suivant les 4 plages de vent local.

- Tri de chacune des bases de données partielles suivant les 4 plages de vent du large.

Les données de surcotes issues de ces tris d'abord suivant les directions de vent et ensuite suivant les vitesses vont nous permettre dans la discussion suivante de tirer certaines conclusions ef poser quelques intérrogations.

\section{DISCUSSIONS}

Le dépouillement des données décrites précédemment conduit aux remarques suivantes:

- Les surcotes calculées à partir de la prédiction marégraphique et des données expérimentales pendant 6 ans (de 1985 à 1990) n'ont pas excédé 65 $\mathrm{cm}$ en surcote et $70 \mathrm{~cm}$ en décote (pendant la tempête d'octobre 1987, le système d'acquisition marégraphique était défaillant: nos données présentent donc des lacunes. Cependant, l'étude ultérieure des tempêtes comblera ce défaut). Pendant cette même période, la vitesse maximale de vent triharaire interpolée à lheure de la basse mer a été de $17 \mathrm{~m} / \mathrm{s}(60 \mathrm{~km} / \mathrm{h})$ à St-Nazaire et, 
de $47 \mathrm{~m} / \mathrm{s}(170 \mathrm{~km} / \mathrm{h})$ au large. Nous verrons par la suite les corrélations entre le vent du large et le vent mesuré à la côte.

- La pression atmosphérique oscille entre 962 et 1046 KPa à St-Nazaire et entre 908 et $1055 \mathrm{KPa}$ au large. On remarque que compte tenu de l'absence de tempêtes, la pression atmosphérique engendre une surcote (ou décote) maximale du même ordre de grandeur que celle due au vent. Cette surcote barométrique a été déduite de la loi du baromètre inversé. Les valeurs de surcotes que nous présentons dans ce document sont corrigées de la pression atmosphérique (Fig. 2)

- De façon globale, lallure des maxima de surcotes et décotes suit celle des maxima de vitesses du vent local. En effet, la figure 2 montre des vents forts enregistrés saisonnièrement (fin d'année et début d'année suivante), ce qui entraine une saisonnarité des surcotes: décotes en hiver et surcotes en été. notamment pour les années 1985,86,87 et 90. Ceci explique pourquol les surcotes sont en général inférieures aux décotes (en valeur obsolue).

- Les études de surcotes faites par TEISSON (1989) avaient conclu que les surcotes maximales ont tendance à se produire par faible coefficient. La figure 3 montre effectivement que toutes les surcotes supérieures a $25 \mathrm{~cm}$ se sont produites par coefficients inférieurs à 80 . Cependant, cette même figure montre que toutes les décotes supérieures à $40 \mathrm{~cm}$ se sont produites par coefficient supérieurs à 75. Dans cette étude de l'influence des coefficients sur les surcotes. nous avons isolé d'une part les marées de revif et d'autre part les marées de déchet. Nous avons alors remarqué que les décotes maximales se sont produites en période de revif.

- Afin de mieux comprendre l'influence de la vitesse et de la direction du vent sur les surcotes et les décotes, nous avons présenté le graphique 3D (Fig. 4 et 5). On s'aperçoit que le vent local présente un aspect de surface beaucoup plus régulier que le vent du large. Sur la figure 4 , on remarque que la surcote augmente avec la vitesse pour le secteur Est à Ouest. La pente de la surface augmente rapidement pour les vitesses supérieures à $8 \mathrm{~m} / \mathrm{s}$. De manière globale, les surcotes maximales se produisent pour des directions comprises entre $W$ et $S W$ et pour des vitesses supérieures à $8 \mathrm{~m} / \mathrm{s}$.

- La figure 5 présente cette surface pour le large. Elle ne présente pas la même régularité que pour la côte. Ceci peut s'expliquer par le fait que l'on dispose de moins de données au large qu'à la côte. L'interpolation y est donc moins bonne. De plus, ces données sont moins représentatives du phénomène car les mesures de vent ne s'effectuent nos al mômo ondrmit 
Partant de ces constatations, selon lesquelles les mesures du vent local sont plus fiables que celles du vent du large, et qu'il existe une corrélation entre le vent local et la surcote (fig. 4), nous reposerons le principal de cette étude à l'exploitation du vent local. Cette corrélation, vent local/surcote parfois réfutée sans preuve est validée ici.

En isolant les paramètres (direction et vitesse) de vent suivant les critères présentés dans la méthodologie nous obtenons une surcote (décote) maximale par secteur de direction. Le découpage en plage de vitesses permet de donner la plage de vitesse de vent (au large et à la côte) soufflant lors de cette surcote (décote).

L'analyse des courbes qui en découlent conduit aux remarques suivantes:

- les surcotes maximales sont plus faibles que les décotes maximales $(f i g, 6)$.

- l'allure générale des surcotes maximales reste la même, que l'on fasse référence au vent local (fig. 6) ou au vent du large (fig. 7): tandis que celle des décotes présente une certaine rotation du large à la côte.

- les décotes maximales se produisent pour un vent local de secteur Nord

- les surcotes maximales, quant à elles correspondent à un vent local de secteur SW (fig. 6).

La lecture de ces figures, appelle cependant à lintérrogation: Pourquoi des vents de mêmes directions et de mêmes vitesses peuvent-ils engendrer aussi bien des décotes que des surcotes ? La remarque faite dans d'autres régions (LACOMBE 1983) selon laquelle une surcote est toujours précédée d'une décote peut-elle répondre à cette intérrogation? C'est ce que nous révélera l'étude ultérieure des tempêtes.

Afin de vérifier si la corrélation surcote/vitesse de vent est plus significative pour un vent local ou un vent du large, nous avons représenté les mêmes résultats que précédemment, mais pour des vents mesurés instantannément à la côte et au large (Fig. 8). Sur la figure 8, les 8 points de mesure sont numérotés afin de suivre la modification des vitesses et directions de vent mesuré instantanément au large et à la côte. Les principales conclusions que l'on peut tirer sont les suivantes: 
- les vents produisant les surcotes maxi viennent d'un secteur S-SW, leur direction demeure identique à la côte et au large. Dans ce secteur le rapport de la vitesse du vent du large à celui de la côte est de 1,2.

- les surcotes maximales produites pour d'autres secteurs ne présentent de corrélation, nien direction. ni en vitesse.

L'étude des vitesses de vent (au large et à la côte) entrainant des maxima de surcotes par plage de direction (suivant les tris décrits précédemment) montre que l'allure des surcotes maximales suit celle des vents maxima aussi bien au large qu'à la côte (Fig. 9). Cette constatation n'avait pas pu être faite pour une surcote quelconque en ce qui concerne le large (Fig. 5).

Nous n'avons pas trouvé de corrélation entre les surcotes maximales (par plage de direction) et les vents correspondants au large. Cependant, nous avons pu établir une relation linéaire entre ces surcotes maximales et les vents locaux correspondants (Fig. 10).

Peut-on extrapoler ce résultat en vue d'une prédiction de la surcote maximale ? Ce qui reviendrait à supposer que la relation est bijective. A priori. nous pensons que ceci peut-être supposé, car la droite indiquée en figure 10. n'est autre que l'enveloppe des surcotes maximales. Actuellement, l'étude de validité de cette relation est en cours.

Une étude identique a été réalisée pour les décotes, mais jusqu'à présent nous n'avons pas pu établir de corrélation. Cependant nous remarquons que les vents correspondants aux décotes sont souvent faibles. Ce quil laisse supposer que, soit la vitesse du vent a une moindre importance, soit qu'il y a décalage entre cause et effet. Actuellement nous sommes en train de rechercher ce décalage.

\section{PERSPECTIVES ET CONCLUSION}

L'analyse des données est certainement l'un des moyens les plus commodes et les plus fiables dans toute étude visant à cerner un phénomène physique. C'est la démarche que nous avons adoptée afin d'étudier les surcotes de marée dues au vent. Le site de l'estuaire de la Loire a été choisi "site témoin" car on y dispose d'une grande quantité de données marégraphiques à Saint-Nazaire et Saint-Gildas, barométriques et anémométriques à SaintNazaire et au larae. 
Le dépouillement des mesures continues dans le temps entre 1985 et 1990 a apporté quelques éléments qu'il nous faudra par la suite confirmer pour d'autres périodes, et dont voici un aperçu:

- les surcotes sont plus faibles que les décotes.

- les décotes maximales se produisent par des vents locaux de secteur Nord, tandis que les surcotes maximales correspondent à un secteur Sud-Ouest.

- une relation linéaire a été trouvée entre les surcotes maximales et les vents locaux.

- etc...

L'analyse des données est toujours en cours, elle s'étendra aux études de tempêtes (vents de plus de $100 \mathrm{~km} / \mathrm{h}$ ) recensées dans ce site depuis les 50 dernières années.

Enfin les résultats généraux de cette étude seront étendus à plusieurs sites de la façade atlantique: Port-Tudy, Le Conquet, le Verdon sur Mer, où nous disposons de données marégraphiques et météorologiques. Ceci permettra certainement de mieux connaitre l'évolution des surcotes dans cette partie du monde.

\section{REFERENCES BIBLIOGRAPHIQUES}

(1) DALSECO S. 1984, "Utiilisation des éléments finis pour le calcul des surcotes", Rapport n³, L.N.H., E41/84.20, E.D.F. Chatou.

(2) DOODSON A.T., 1954, "Développement harmoniqe du potentiel générateur de la marée". Revue Hydrographique Internationale, pp : 29-6].

(3) GHADRI H., O.A. KHALIFA M.A., GROVEL A., 1990, "Prévision des surcotes dans l'estuaire interne de la Loire par une approche statistique". Premières journées Inter-Universitaires Génie Civil-Génie Côtier, Le Havre, pp : 75-86.

(3) GHADRI H., O. A. KHALIFA M. A., GROVEL A., 1991, "Etude phénomenologique des surcotes dues au vent". Colloque Le Littoral Ses Contraintes Environnementales Et Ses Conflits D'Utilisation. Nantes.

(4) LACOMBE, 1983, "Le grand atlas de la mer", Encyclopaedia Universalis Albin Michel.

(5) SHOM, 1985. Table de marées des grands ports du monde, Paris. 
(6) TEISSON Ch., 1984, "Etude statistique de surcotes pour le site du Carnet", Rapport L.N.H., HE/42/84.13, E.D.F. Chatou.

(7) TElsSON Ch. 1989, "Prise en compte de la tempête du 16 octobre 1987 dans l'étude statistique des surcotes au Carnet", Rapport L.N.H., HE-42/88.30, E.D.F. Chatou.

(8) VRIJLING J.K. , BRUINSMAJ. , 1990, "Hydraulic boundary conditions related to the design of the Oosterscheld storm surge Barrier in the Netheriands. An example of adjoint distribution of waves and surges"; Coastal Protection. Pilarezyk(ed), Balkema, Rotterdam, pp: 423 - 446.

\section{REMERCIEMENTS}

Nous remercions le Port Autonome de Nantes/St Nazaire et METEO FRANCE, d'avoir mis à notre disposition respectivement les enregistrements marégraphiques et les données météorologiques à St Nazaire. 

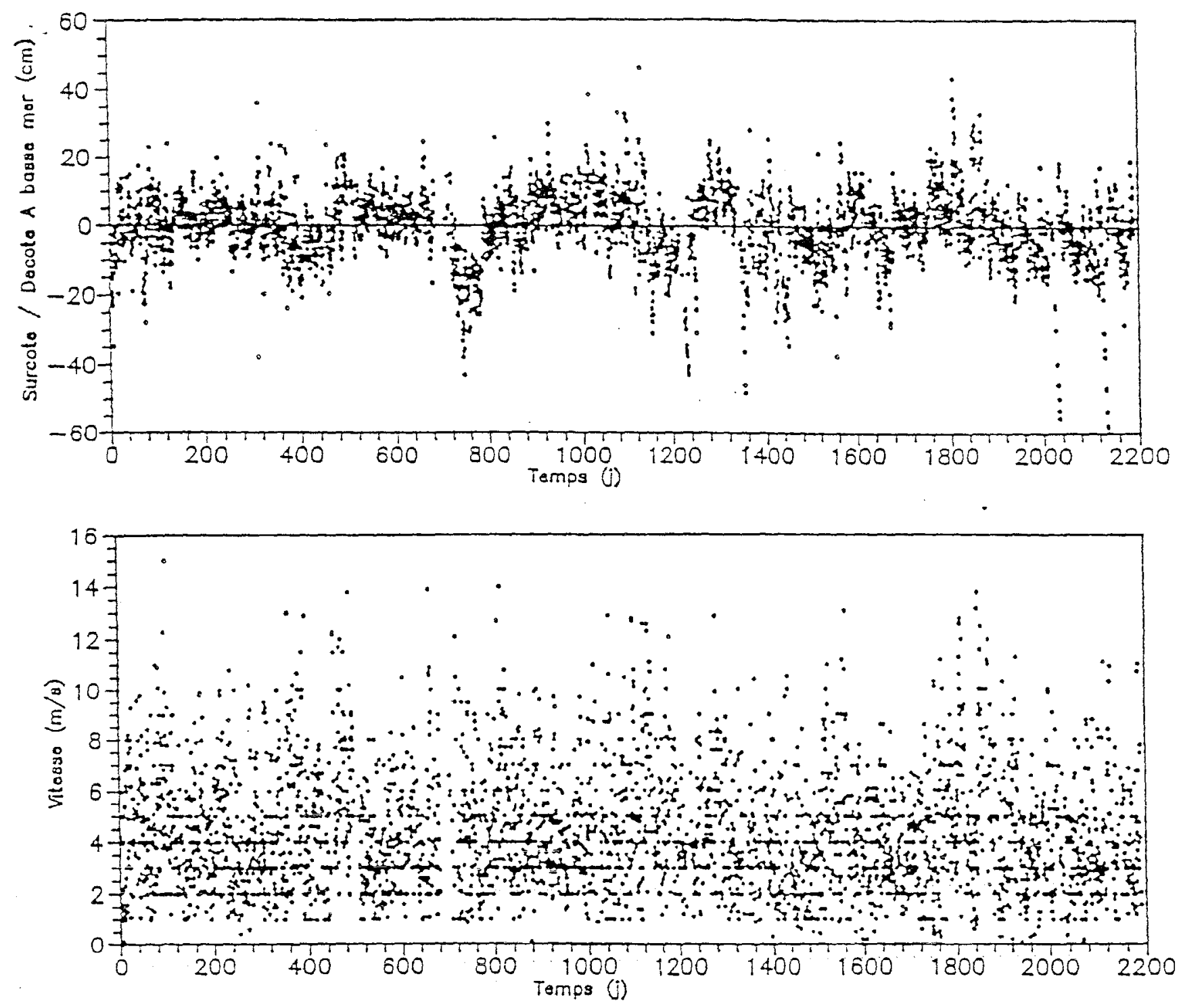

Fig. 2 : Surcotes (Décotes) et vitesse de vents en fonction du temps St-GILDAS. du 01/01/1985 au 31/12/1990

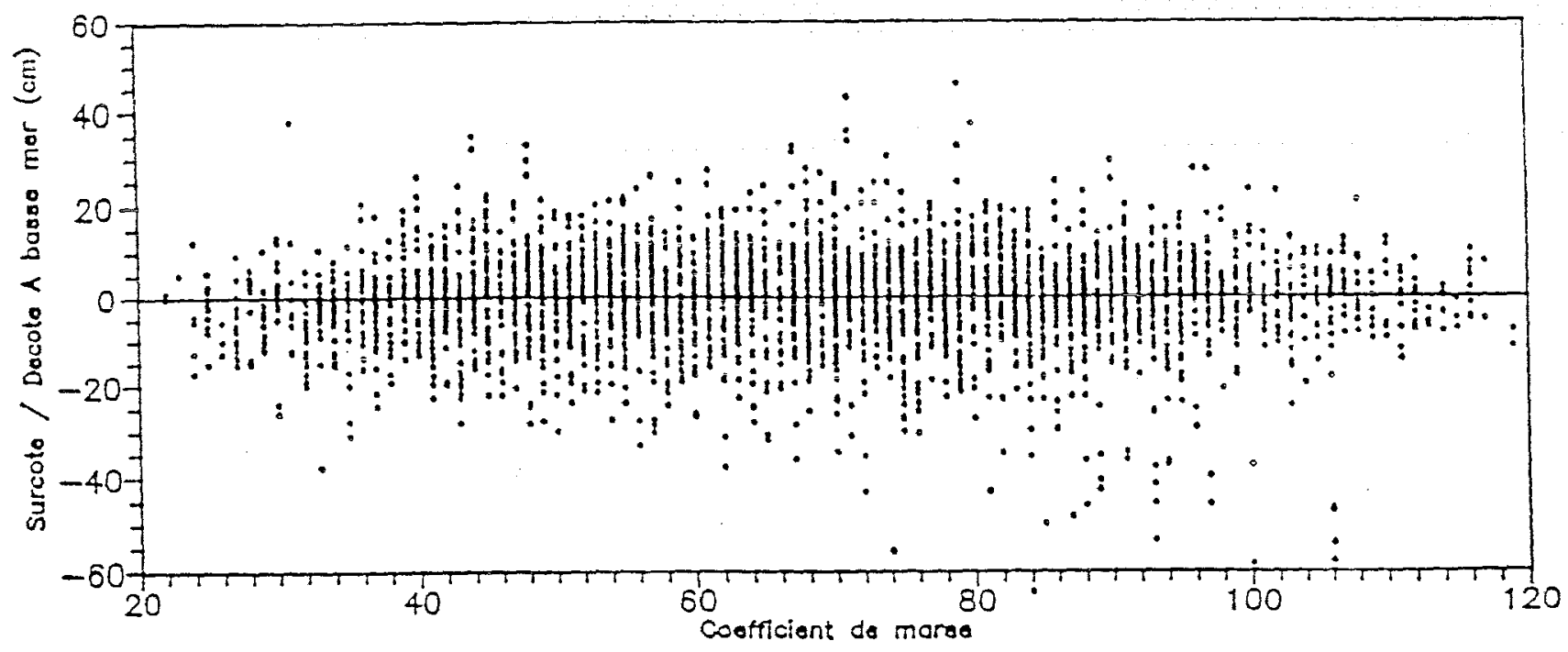

Fig. 3 : Surcote (Décote) en fonction des coefficients de marée St-GILDAS. du 01/01/1985 au 31/12/1990 


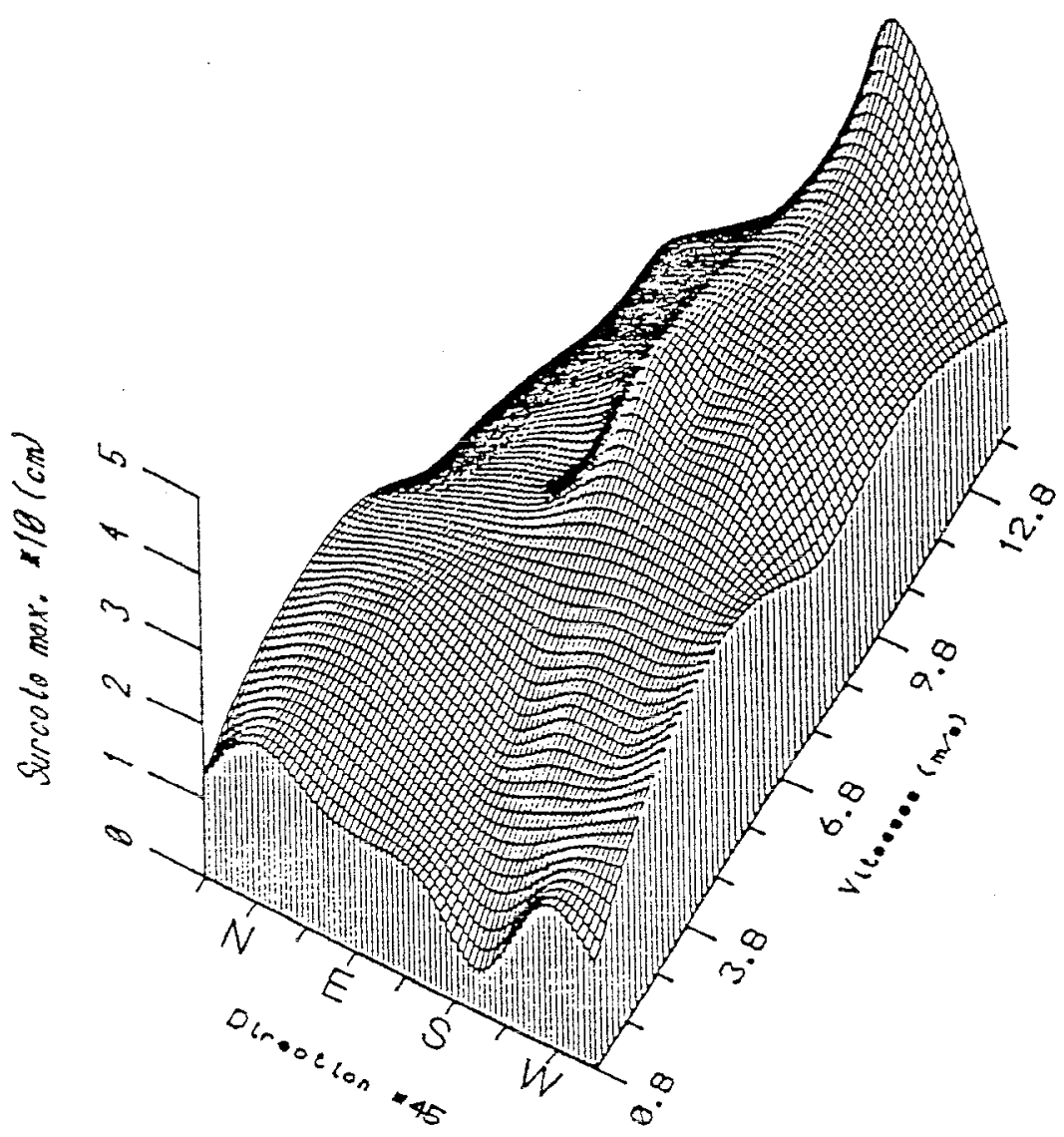

Fig. 4 : Sur. max. en fonction des Dir. et des Vit. de vents locaux à St-GILDAS

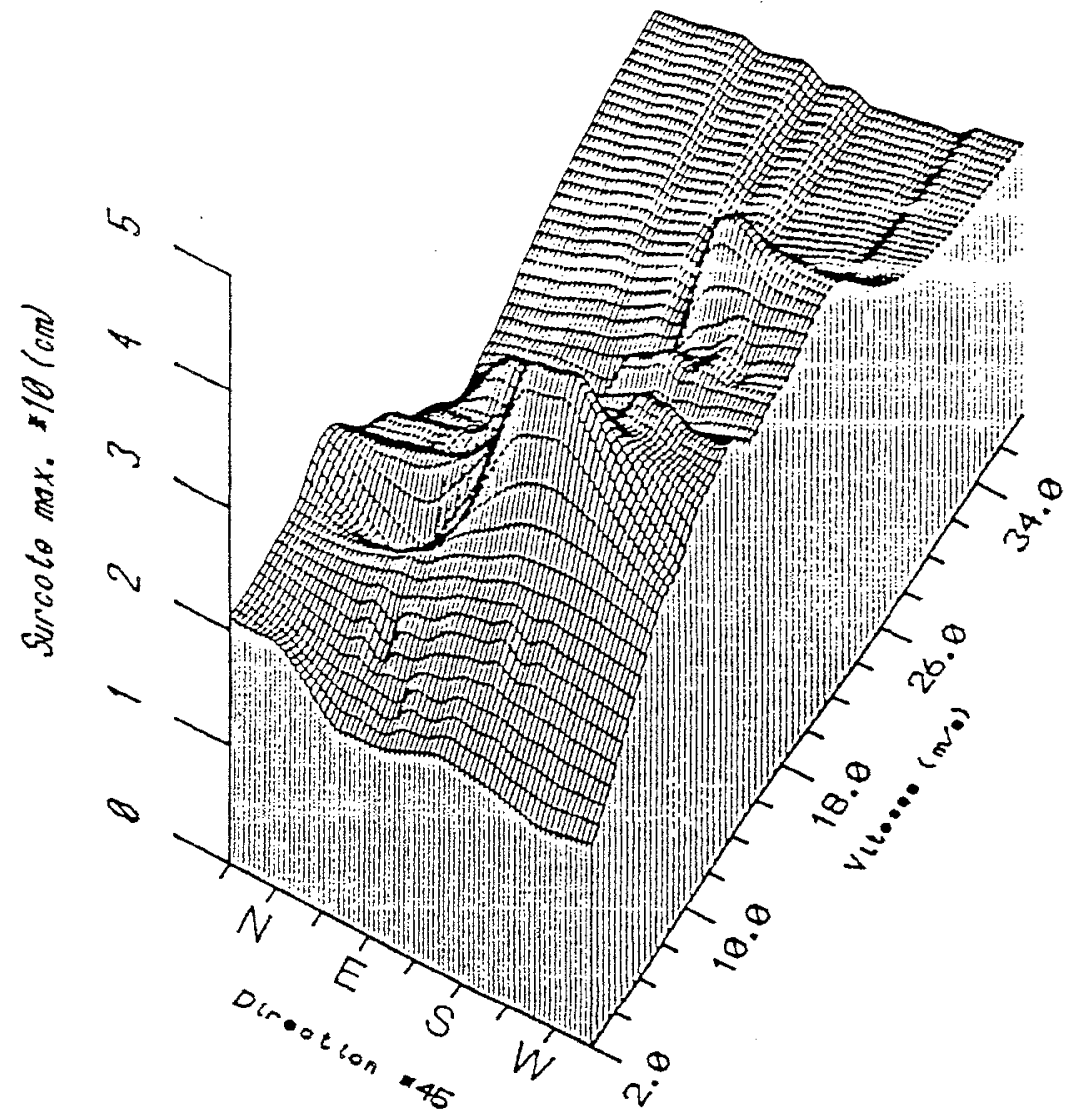

Fig. 5 : Sur. max. en fonction des Dir. et des Vit. de vents au large 


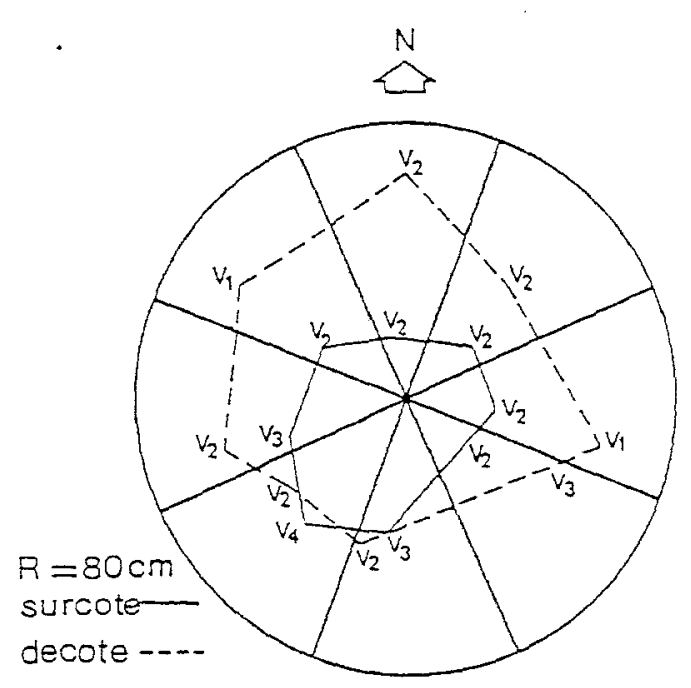

Fig. 6: Maxima des surcotes et décotes Fig. 7 : Maxima des surcotes et décotes à basse mer à St-Gildas, liées à la à basse mer à St-Gildas, liées à la direction et à la vitesse de vent à la direction et à la vitesse de vent au large

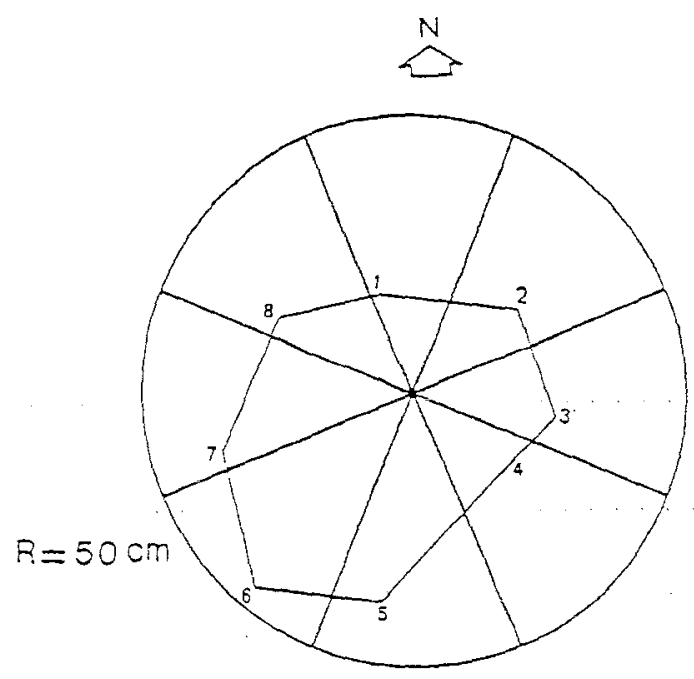

(a)

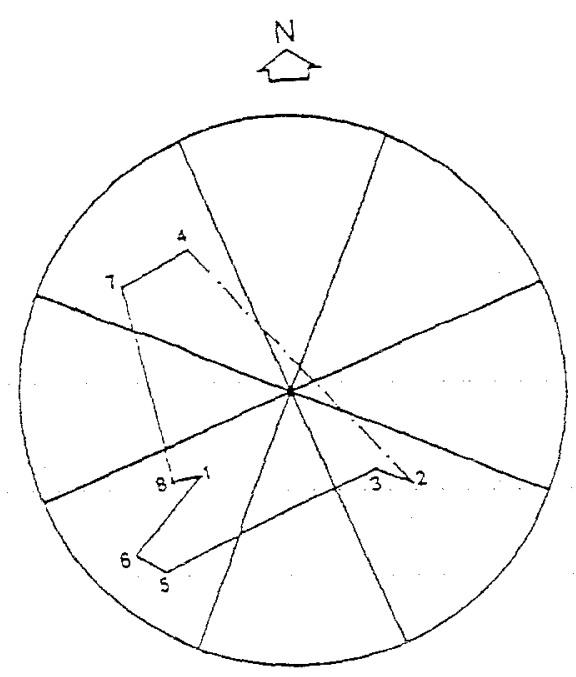

(b)

Fig. 8 : Maxima des surcotes à basse mer à St-Gildas, liées à la direction et à la vitesse de vents instantanées à la côte (a) et au large (b)

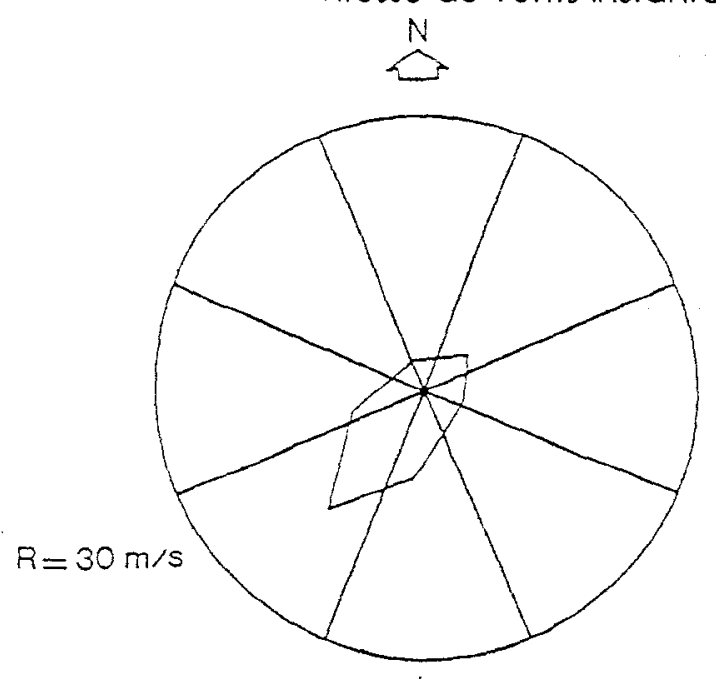

(a)

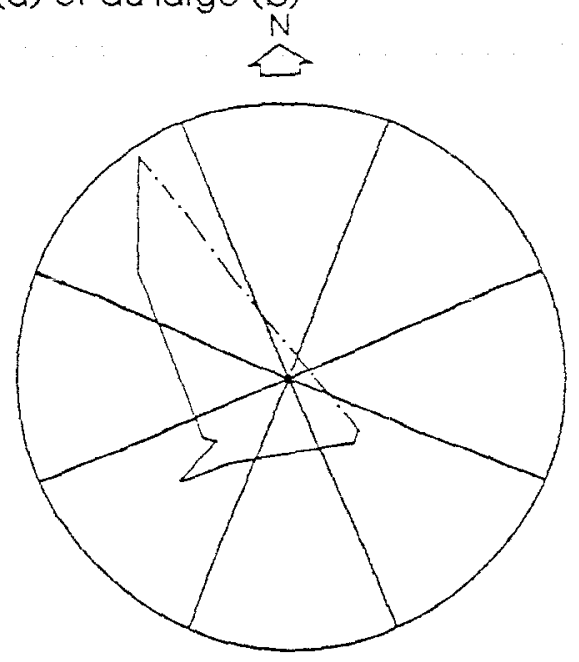

(b)

Fig. 9: Vitesse de vent instantané à la côte (a) et au large (b) produisant les maxima de surcotes 


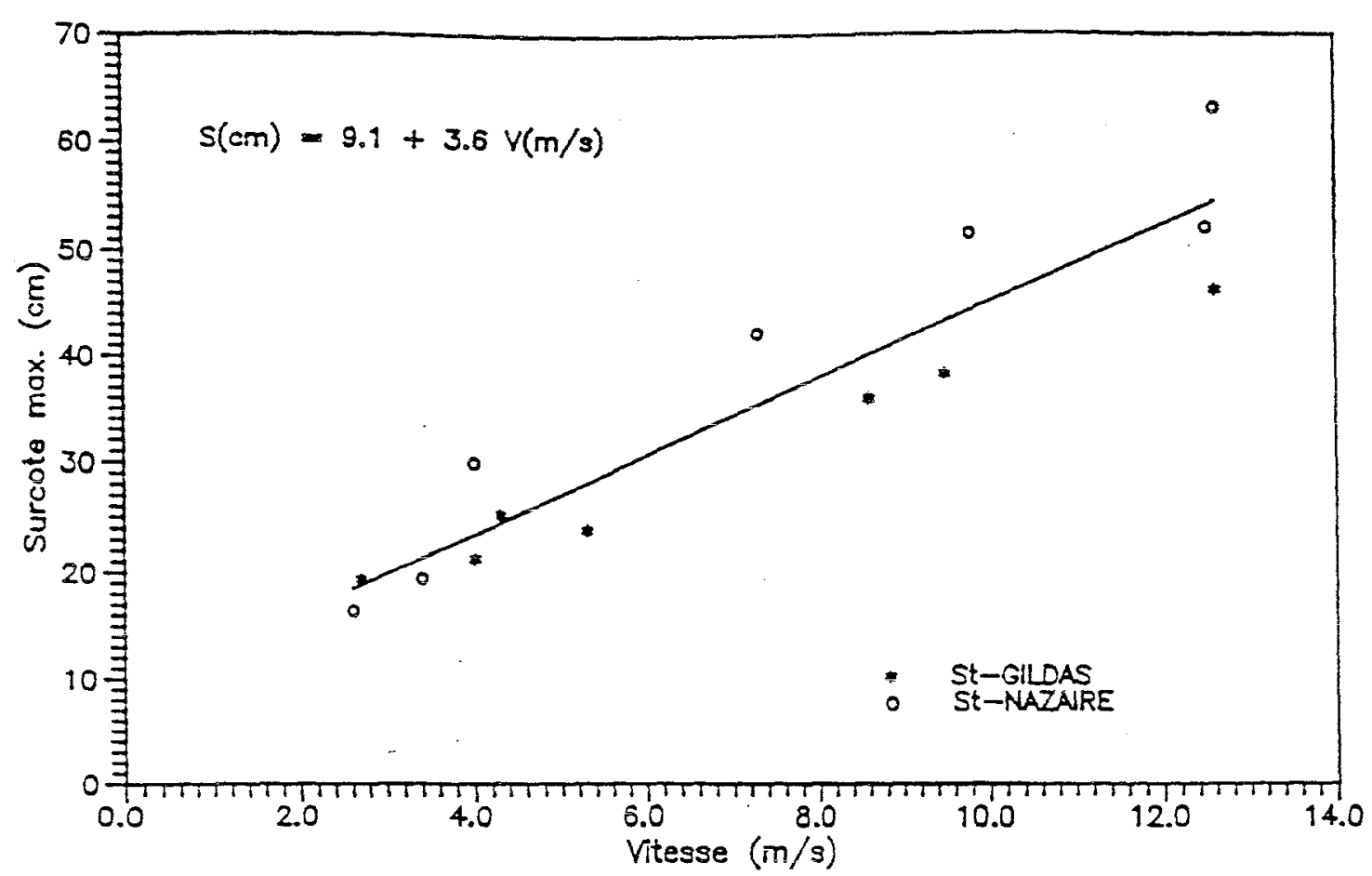

Fig. 10: Correlation entre les surcotes maxiamles (par plage de direction) et les vitesses de vents correspondants à la côte 